Chapter 9

\title{
Mechanisms and Treatment of Photoaging and Photodamage
}

\author{
Marianna Kulka \\ Additional information is available at the end of the chapter \\ http://dx.doi.org/10.5772/56425
}

\section{Introduction}

Damage caused by excessive, long-term sun exposure contributes to signs of ageing and is most readily recognizable on our external surface, the skin. The damaging effects of the sun are mainly caused by ultraviolet radiation (UVR) which, on earth, consists of UVA (320 to $400 \mathrm{~nm}$ ) and UVB (280 to $320 \mathrm{~nm}$ ). The third type of UV radiation, UVC, also called shortwave or ionizing radiation, is absorbed by gasses in our atmosphere, does not reach the earth's surface and does not normally contribute to photodamage of skin. Ozone is one of the gases that absorbs UVC - fortunately, since UVC's ionizing effects can significantly alter cellular structures and molecules. Although UVB and UVA radiation does not have enough energy to ionize atoms, it can alter chemical bonds in molecules, thus altering their structure and functions. Skin cells are particularly susceptible to alterations mediated principally by the creation of free radicals and oxidative stress and changes in DNA structure can result in constitutive expression of protocongenes and ultimately the development of cancerous skin lesions.

Through its changes of molecular structures, UV radiation causes a series of biochemical and structural changes in skin tissues. Some of these effects, such as the induction of vitamin D production, are actually very beneficial to skin health. Niels Ryberg Finsen, a Danish physician and scientist, was one of the first to show that light can be both beneficial and detrimental to human skin and his works Om Lysets Indvirkninger paa Huden ("On the effects of light on the skin"), published in 1893 and Om Anvendelse i Medicinen af koncentrerede kemiske Lysstraaler ("The use of concentrated chemical light rays in medicine"), published in 1896 created the paradigm on which photoaging and photodamage research was based for the next fifty years. Finsen won the Nobel Prize in Physiology in 1903 for his work on phototherapy in which he showed that certain wavelengths of light (UV in particular) could treat lupus 
vulgaris, cutaneous tuberculosis. However, UV radiation can also initiate some deleterious effects that fundamentally change the structure and function of the skin and its constituent components. In fact, it has been shown that repeated, excessive sun exposure can initiate and perpetuate an inflammatory response that, given time, causes breakdown of the skin's immunological functions.

This review will focus on the effects of sun exposure on the skin microenvironment - particularly the structural proteins and immune cells that are key players in the skin's protective functions. There is sufficient evidence to suggest that photodamage is associated with a low level of chronic inflammation that ultimately breaks down the skin's structure and results in some of the manifestations of skin aging. Consequently, skin treatments that target these processes are primarily focused on rebuilding the skin's architecture and promoting its biochemical regeneration. While some treatments have been found to have some beneficial effects to the underlying cellular immune systems regulating skin inflammation and regeneration, others may, in fact, be deleterious to these processes. In this review, I will discuss some naturally-derived bioactive compounds and formulations that have been shown or are suggested to be effective at modulating the pathophysiological changes associated with photodamage and photoaging. Bioactive compounds that can address these immunological changes are uniquely poised to not only reduce the damage associated with photodamage but may ultimately improve the resiliency and protective nature of the skin surface.

\section{Skin is a protective organ}

\subsection{The three layers of defense}

Healthy human skin is an important physical barrier to the environment and protects the body from a variety of insults. It is the largest human organ and comprises approximately 15 percent of a person's body weight and covers about 1.5 to $2.0 \mathrm{~m}^{2}$ of our surface area. Skin is mainly composed of water (70\%), protein (25\%) and lipids ( $2 \%)$ forming an effective barrier to dehydration, pathogens and mechanical insults such as abrasion. In a mechanical sense, the main function of the skin is to serve as a protective barrier to keep good things in (water, nutrients and heat) and keep bad things out (pathogens, UV radiation, toxins).

In order to understand the essential functions of the skin, it is important to review the complexity of this large organ. The skin is composed of three main layers, the uppermost epidermis, the lower dermis and the hypodermis (Fig. 1). Each of these layers is composed of a specific set of cells that perform an essential function in that layer. The epidermis contains keratinocytes, melanocytes and Langerhans cells which are critical for the structural and functional integrity of the epidermis. Keratinocytes are the major population of cells and originate in the bottom-most stem-cell pool in the stratum spinosum (Fig. 1). The epidermis itself is composed of five layers: stratum corneum, stratum lucidum, stratum granulosum, stratum spinosum and stratum basale. The uppermost layer of the skin, the keratinous stratum corneum, renews itself every 3-5 weeks and is principally composed of dead keratinocytes (see section below on keratinocytes for more detail). Keratinocytes have the capacity to 
renew themselves every 4 weeks but this process can be increased during injury or inflammation. The underlying dermis is a vascularized, connective tissue containing nerve endings, glands and lymphatic vessels and provides structural and nutritional support to the upper epidermal cells. The dermis is, in fact, the a mucopolysaccharide matrix composed of collagen and elastin fibers. In this layer, there is a great variety of cells including mast cells, fibroblasts, macrophages and Langerhans cells all of which contribute to the immune response observed in some skin pathologies such as atopic dermatitis. These cells also maintain and regulate the essential healthy functions of skin such as repairing injury, preventing infection, regulating circulation, preventing dehydration and providing nutrition. Beneath the dermis is the superficial fascia, or hypodermis, which is comprised primarily of fat tissue.

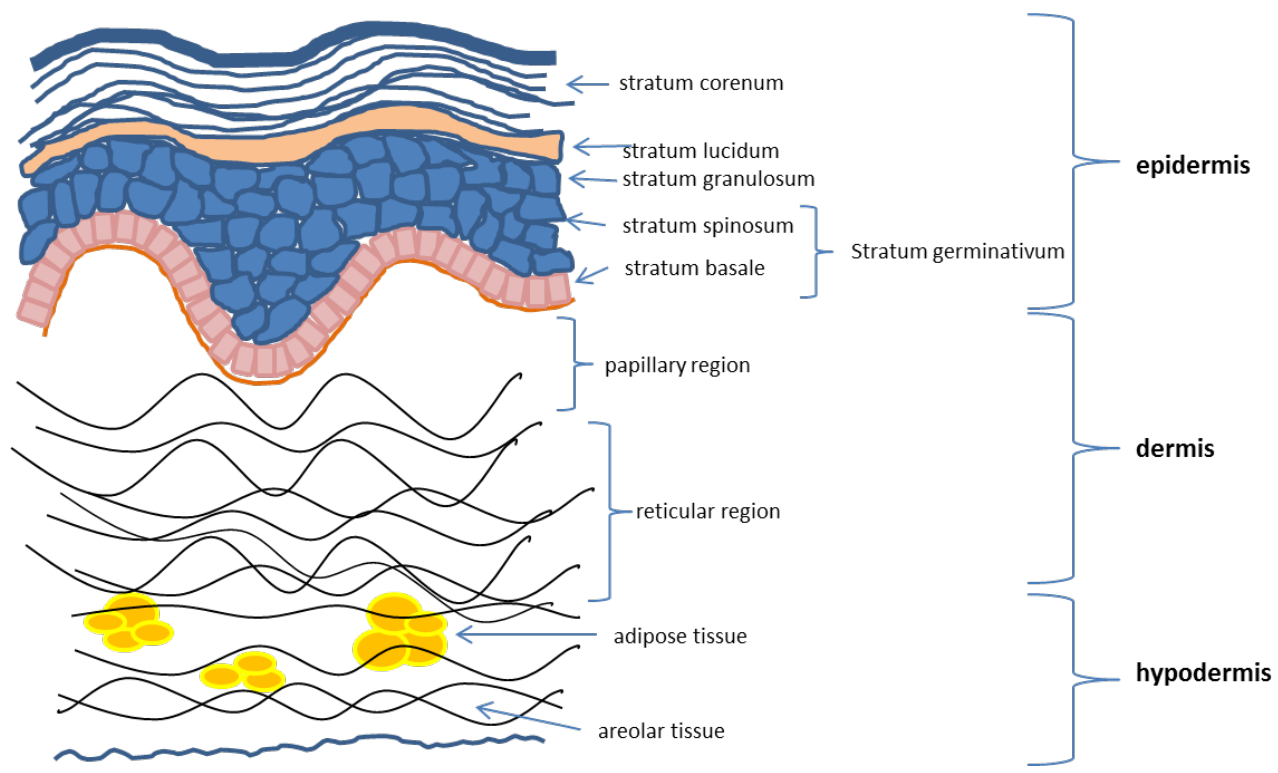

Figure 1. Structure of skin. Skin is comprised of three main layers as shown. The epidermis contains keratinocytes, melanocytes and Langerhans cells which are critical for the structural and functional integrity of the epidermis. The epidermis itself is composed of five layers: stratum corneum, stratum lucidum, stratum granulosum, stratum spinosum and stratum basale. The strata granulosum, spinosum and basale all contain keratinocytes whereas the strata corneum and lucidum have only dead keratinized cells. The underlying dermis is a vascularized, connective tissue containing nerve endings, glands and lymphatic vessels. Beneath the dermis is the superficial fascia, or hypodermis, which is comprised primarily of fat tissue.

\subsection{Melanin and melanogenesis - A protective response}

As a result of its optical properties, the stratum corneum scatters photons and reflects UV radiation, providing some degree of protection. However, a portion of UV radiation penetrates this uppermost layer and can potentially damage the delicate structures beneath. Mel- 
anin plays an important role in the skin's function as a protective barrier by absorbing UV radiation and thus protecting the underlying cells from DNA damage. The word melanin comes from the Greek word melan, meaning black. Melanin is a polymeric pigment derived from tyrosine and, in humans, it is synthesized in two main forms: the brown/black eumelanin and the cysteine-rich red/brown pheomelanin. The chemical structures of these two types of melanin are different resulting in different properties and functions (Fig. 2). Whereas eumelanin is composed of dihydroxyindole carboxylic acids, pheomelanin is a polymer of benxothiazine units. The carboxylic structure may account for pheomelanin's weak shielding capacity against ultraviolet radiation relative to eumelanin. In fact, pheomelanin has been shown to amplify UVA-induced reactive oxygen species (ROS)[1]. For this reason, pheomelanin is sometimes referred to as a UV-sensitizer and in some instances has been considered to have a weak carcinogenic effect in terms of melanoma formation.<smiles>CC1=c2cc(C(=O)O)[nH]c2=C(c2c(O)c(O)c(C(=O)c3cc(C(=O)O)[nH]c3C(=O)O)c3[nH]c(C(=O)O)cc23)C(=O)C1=O</smiles>

Eumelanin

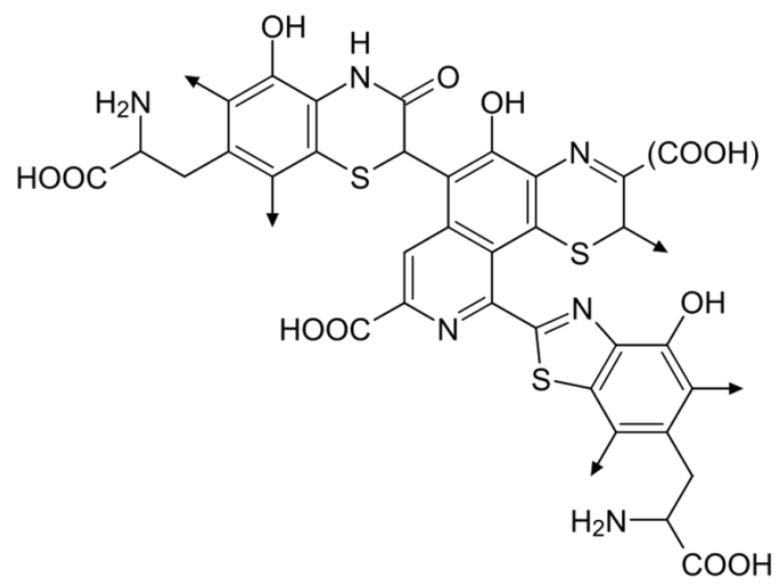

Pheomelanin

Figure 2. Chemical structure of eumelanin and pheomelanin.

Melanogenesis refers to the biochemical process by which melanin is formed. Melanocytes are the principal cell type responsible for the production and distribution of melanin in the skin. Since melanocyte differentiation and proliferation is linked very closely with their ability to produce melanin, melanogenesis is sometimes used to describe the process of melanocyte viability and reproduction. Indeed, many cosmetic products on the market that profess to alter or manipulate melanogenesis are actually cytotoxic to melanocyte and by killing healthy melanocytes they indirectly decrease the amount of melanin in the human epidermis. For example, hydroquinone (HQ), which is an U.S. Food and Drug Administration (FDA) -approved product, is used in $2-4$ percent concentration as a skin-lightening agent but is considered to be cytotoxic to melanocytes[2]. Interestingly, some antibiotics used to 
treat ear infections, such as amikacin, are cytotoxic to melanocytes as well and one of its principal side-effects is ototoxicity[3].

One of the most obvious and controversial side-effects of exposing our skin to UV radiation is an increase in skin pigmentation. Certainly the issue of tanning and tanning beds has recently gained a great deal of attention because it has been shown that exposure to prolonged UV radiation (either from tanning beds or outside exposure) can cause melanoma and an effort is underway to limit the amount of time that people, especially children, spend tanning in any setting. The process of tanning is a protective mechanism and the skin's way of protecting its internal biochemical reactions from UV damage. Increased pigmentation is caused by increased production of melanin which is able to absorb some UV radiation and thus protect more sensitive biological pathways in the cell[4]. Epidemiological studies have shown that people who have high levels of constitutive pigment in their skin and are able to "tan well" are less likely to develop skin cancer[5], [6].

We still do not fully understand the precise mechanisms that initiate the malignancies associated with skin cancer and we certainly have an incomplete understanding of how photodamage and repair functions in people of different skin colors. However, mouse models and human studies have shown that albinos have a lower incidence of melanoma than their fair skinned counterparts[7] suggesting that melanin cannot just function as a protective UV-energy absorber. Recently, several groups have shown that melanin, especially pheomelanin (a yellow/red form of melanin), acts as a potent UVB photosensitizer to induce DNA damage and cause apoptosis in mouse skin. These groups believe that pheomelanin contributes to UV-induced DNA damage that is incompletely repaired. They further believe that this DNA damage maps to specific sequences of BRAF and N-RAS genes, both of which are frequently mutated in human melanoma[8], [9].

Some organic and inorganic compounds mimic the effects of melanin by absorbing or scattering UVR due to their particulate nature - often in the nanoscale. These chemicals are processed and made available as lotions, sprays, gels or other topical applications commonly known as sunscreens. There are 17 different chemicals that have been approved by the U.S. FDA as active ingredients in sunscreens. Oxybenzone (benzopehone-3) is the most widely used sunscreen chemical worldwide but other chemicals include octinoxate, avobenzone, and octyl salicylate. Some of the physical sunscreens are micro or nano-ionized to create particulate formulations capable of forming a protective UV barrier on the skin's surface. Titanium dioxide $\left(\mathrm{TiO}_{2}\right)$ are often used as a inorganic protective sunscreens in cosmetic products and to adjust their protective factor (SPF), they are often coupled with organic chemicals such as p-amino benzoic acid (PABA) which absorbs UVB but not UVA radiation. Recently, some have suggested that these nanoparticles (NP; around $200 \mathrm{~nm}$ ) may penetrate the skin and modify the skin's immune-environment. However, several groups have shown that although nanoparticles are unable to penetrate healthy, intact human epidermis[10] they can significantly disturb cell functions through direct contact when the skin barrier is compromised[11], [12]. In fact, NP can reach the epidermal layer via hair follicles in certain circumstances such as laser-treatment[13] or inflammation[14], [15] but most compounds, although targeting the follicular region, do not permeate past the pilosebaceous region[16]. 


\section{The production of melanin}

\subsection{Tyrosinase}

Tyrosinase is responsible for the hydroxylation of monophenol and conversion of an $o$-diphenol to the corresponding $o$-quinone which then undergoes several modifications to become melanin (Fig. 3). Tyrosinases are ubiquitously expressed by both plants and animals, and although they may vary in structure, they all contain copper as an important cofactor. Human tyrosinase is a transmembrane protein and is sorted into specialized organelles in melanocytes called melanosomes such that melanin is ultimately synthesized and stored within these melanosomes[17]. Tyrosinase is an excellent example of convergent evolution in that although both animals and plants express tyrosinase, and although tyrosinase essentially performs the same function in all of these organisms, the structure and enzymatic requirements of these proteins are diverse. Regardless, tyrosinase regulates both the type and amount of melanin synthesized in the specialized cells that contain it. In humans, tyrosinase is encoded by the tyr gene which when mutated causes albinism in humans[18]. In Caucasians, tyr mutations can be identified in $56 \%$ of patients with autosomal recessive ocular albinism, characterized by reduced pigmentation of the eye[19].

\subsection{Microphthalmia-Associated Transcription Factor (MITF)}

Transcription of tyr is controlled by microphthalmia-associated transcription factor (MITF). MITF acts both as a transcription activator to promote expression of genes involved in melanogenesis within the cell cycle and as a transcriptional repressor of genes involved in invasion, making elevated MITF levels a possible biomarker for melanoma[20]. In humans, MITF regulates the expression of tyrosinase (TYR), tyrosinase-related protein-1 (TRP-1), and tyrosinase-related protein-2 (TRP-2). A complex regulatory network precisely modulates MITF expression and activation and therefore controls tyrosinase and melanogenesis. Therefore, targeting only tyrosinase or only MITF is destined to be either an ineffectual or a temporary intervention. It is likely that combination therapy that targets MITF, tyrosinase and the other regulatory pathways is more likely to produce a measurable and prolonged effect on skin pigmentation.

One of the key pathways in the stimulation of melanocytes in response to UV radiation is the adenylate cyclase pathway. UV radiation mediates the synthesis and release of alphamelanocyte-stimulating hormone $(\alpha-\mathrm{MSH})$ which then promotes pigmentation by binding to the melanocortin 1 receptor (MC1R) on the surface of melanocytes. In people with the red-hair/fair-skin phenotype, the MC1R gene is non-signaling which leads to the increased risk for the development of melanoma. The production of cyclic adenosine monophosphate (cAMP) downstream of MC1R activates the receptors of MITF. MITF then participates in the conversion of tyrosine into melanin pigments by stimulating the transcription of melanocyte-specific genes. Therapeutic agents that are able to inhibit MITF through the down regulation of MC1R activity are likely to be particularly effective in reducing melanocyte activity. 


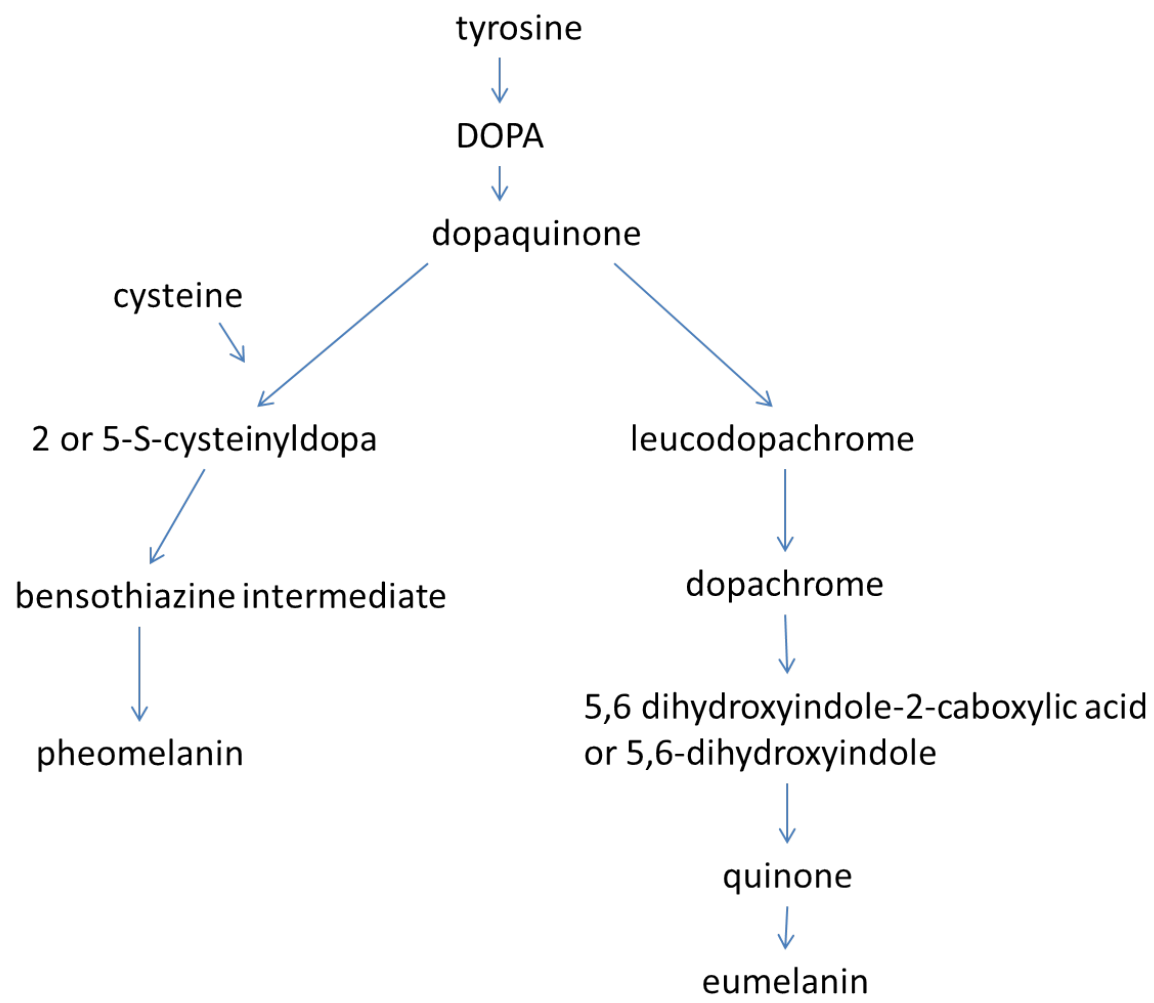

Figure 3. Melanin biosynthesis.

\subsection{Keratinocytes}

Keratinocytes are an important structural cell of the epidermis and comprise the majority of the cells in this layer of the skin. Throughout their life cycle, keratinocytes can take on many forms. Keratinocytes are produced by mitosis in the most basal layer of the epidermis when they begin to produce a great deal of keratin. Eventually, older keratinocytes are pushed up to the upper layers by the rapidly dividing keratinocytes beneath them until they reach the stratum granulosum where they begin to drastically change their morphological and biochemical functions. In the stratum granulosum, keratinocytes lose their organelles and acquire a flattened appearance. Due to their production of keratin, these cells become strong and their tightly bound structure makes the outermost keratinocyte layer strong and relatively impermeable.

An important role of keratinocytes is the transport of melanin through the layers of the skin. Once melanin is produced by melanocytes, the pigments are packaged into melanosomes that are subsequently translocated along the dendrites and then captured and aggregated by 
keratinocytes. The mechanism of transfer has long been a mystery, however a recent study summarizes the transfer mechanism in three steps: (i) the melanocyte's dendrite attaches to the surface of the keratinocyte, (ii) a portion of the melanocyte's dendrite sheds in order to deposit the melanosomes on the surface of the keratinocyte, (ii) and finally, the keratinocyte engulfs the melanosome package[21].

\section{Natural ingredients that reduce pigmentation}

Since a number of naturally derived bioactive molecules have been found to reduce skin pigmentation, the cosmetic industry has focused on the development of these compounds for use in their products. These compounds inhibit the production of melanin at different stages of melanogenesis. There are several compounds that specifically inhibit tyrosinase. For example, terrein, a bioactive fungal metabolite agent isolated from the Penicillium species, has been shown to strongly decrease the production of tyrosinase by down-regulating MITF through dual pathways[22]. One pathway of these pathways may involve the activation of extracellular signal-regulated kinase (ERK) which has been reported to reduce melanin synthesis[23]. A second pathway recently identified may involve the ubiquitination and subsequent breakdown of tyrosinase itself[24].

Flavonoids, in particular, have received a great deal attention for their potential pigment-reducing activity because they are seen as "natural" and are associated with less side-effects than conventional medications and synthetically-designed compounds. Flavonoids are polyphenolic compounds typically found in plants. These compounds are ideal candidates for cosmetic purposes because of their potent bio-activities and low toxicity. Aloesin, a compound isolated from aloe extracts, has been shown to successfully reduce tyrosinase in vitro by inhibiting the hydroxylase activities[25], [26]. Resveratrol, a compound found in red wine, appears to have an affinity for tyrosinase and, by an unknown mechanism, reduces tyrosinase activity and MITF expression[27] although some studies have questioned its ability to reduce tyrosinase expression when used alone[28]. Flavonoids isolated from licorice are able to inhibit mushroom tyrosinase[29] and a skin lightening agent that uses licorice extract in its formulation (comprised of many bioactive ingredients) has been shown to reduce pigmentation in patients[30]. There are many reports of flavonoids from various sources interfering with or reducing the activity of tyrosinase, and although they all show some efficacy at reducing the biochemical pathways that lead to pigmentation, it is still unclear whether targeting these pathways in human skin would necessarily lead to reduced melanin formation in vivo.

Melanin formation is a complex process and there are compounds that may inhibit melanogenesis without necessarily inhibiting tyrosinase function. Anti-oxidants such as fermented rice bran and vanillic acid can reduce expression of MITF, MC1R, and associated biochemical pathways, without affecting tyrosinase activity,[31], [32] possibly due to their ability to impair peroxidase activity. Anti-oxidants, in general, may potentiate the MC1R pathway which itself is able to reduce downstream levels of hydrogen peroxide and promote nucleo- 
tide excision repair mechanisms in response to UV radiation[33]. The process of melanogenesis involves cell-to-cell communication between keratinocytes and melanosomes and compound such as niacinamide, a bioactive form of niacin (vitamin B3), has been shown to reduce pigmentation both in vitro and in vivo by downregulating the amount of melanosomes transferred to keratinocytes[34].

\section{Photoaging and skin cells}

\subsection{Photoaging}

As skin ages, a number of changes occur in the structure of the connective tissues. Healthy human dermis consists primarily of an extracellular matrix and a fibrous component formed by collagen and elastic fibers. The complex network of collagen accounts for approximately 80 percent of the dry weight of the skin and it is the component that provides the resistance to deformation. Elastic fibers comprise 2 to 4 percent of the dry weight of the skin and provide elasticity. Glycosaminoglycans (complex sugars, including hyaluronic acid) and water provide the viscous component of the deformation itself. As skin undergoes normal aging, a general reduction in the production of collagen fibers and proteins that make up the extracellular matrix contributes the formation of wrinkles and flaccid skin. Similarly an increased rate of degeneration of elastic fibers leads to the loss of elasticity.

Many of the changes that occur in skin structure as a result of aging are expressed prematurely in photodamaged skin, a phenomenon known as photoaging. Photoaging refers to the various cellular processes associated with senescence that result from photodamage. These processes are characterized by two events referred to as intrinsic (chronoaging) and extrinsic (photoaging) ageing. Leveque et al. first described the effects of chronic sun exposure on the mechanical properties of the skin and showed that long term sun exposure caused a decrease in skin extensibility and elastic recovery[35]. The skin also appeared thicker, more rigid and less susceptible to deformation. Berardesca and Maibach confirmed these findings using controlled ultraviolet (UVA) treatment and showed that even short term, low UVA and UVB exposure can impair skin elasticity and that inflammation and edema are characteristic of this process[36]. Other clinical features of photoaging include wrinkles, dyspigmentation, yellow hue, laxity, vascular ectasia, and malignancies. Adult populations in European and North American countries experience a prevalence of photoaging as high as 80 to 90 percent.

\section{Photodamage and impact on skin cells}

\subsection{Photodamage}

Photodamage refers to the damage of human skin by ultraviolet light, specifically UVA and UVB, and although the detailed mechanisms of photodamage are not fully understood, 
some general features have been described and are used as a standard by which topical treatments are judged. Physiological consequences of UVA radiation include thickening of the stratum corneum, epidermal hyperplasia, depletion of Langerhans cells, and dermal inflammation. Although it may seem as if these changes are random and entirely dependent upon UV exposure, photodamage is, in fact, a tightly controlled and regulated process that is facilitated by specialized skin cells. The ways in which these cells interact with each other and their extracellular matrix ultimately determines the morphological and histological consequences of light exposure in the skin. The cells and proteins involved in this process are numerous and the connections between them are complex. However, exposure of the skin to UVR initiates oxidative stress and triggers the synthesis of matrix metalloproteases (MMP) [37]. The production of MMP results in the degradation of dermal collagen and other matrix molecules thus reducing the elasticity and integrity of the skin[38].

\subsection{Keratinocytes}

Since keratinocytes in the upper layers of the epidermis are dead and do not underdo mitosis or metabolisis, they are relatively resistant to UVR induced damage. Younger, keratinocytes located deeper in the epidermis are much more susceptible to UVR damage and repeated UVR exposure results in accumulated DNA mutations that can lead to epidermal malignancies. Keratinocytes play a central role in elaborating innate responses that lead to inflammation and influence the generation of adaptive immune responses in skin.

Apart from the minor cellular constituents of the epidermis, specifically Langerhans cells and melanocytes, keratinocytes are the major source of cytokines. UV radiation exposure stimulates keratinocytes to secrete abundant pro-inflammatory IL-1-family proteins, IL-1 $\alpha$, IL-1 $\beta$, IL-18, and IL-33. Normal skin contains only low levels of inactive precursor forms of IL-1 $\beta$ and IL-18, which require caspase 1-mediated proteolysis for their maturation and secretion. However, caspase- 1 activation is not constitutive, but dependents on the UV-induced formation of an active inflammasome complex. IL-1 family cytokines can induce a secondary cascade of mediators and cytokines from keratinocytes and other cells resulting in wide range of innate processes including infiltration of inflammatory leukocytes, induction of immunosuppression, DNA repair or apoptosis. Thus, the ability of keratinocytes to produce a wide repertoire of proinflammatory cytokines can influence the immune response locally as well as systematically, and alter the host response to photodamaged cells.

\subsection{Fibroblasts and langerhans cells}

The most common type of cell found in the connective tissue of skin is fibroblast. They assist in producing and organizing the extracellular matrix of the dermis and also communicate with each other and other cells in the regulation of skin physiology. Dermal fibroblasts are a heterogeneous population and are identified by their location within the dermis. Fibroblasts that exist in the papillary dermis are generally in a more active state and divide at faster rates than those that exist in the reticular layer of the dermis[39]. Fibroblasts produce and secrete precursors of the extracellular matrix such as collagen, glycoproteins, and other molecules that support the structure of the skin. 
Studies have shown that when exposed to UVA, fibroblasts proliferative activity is slowed or stopped due to morphological and functional changes. When exposed to sublethal UVA radiation does in vitro, researchers observed mutations in the mitochondrial DNA (mtDNA) in fibroblasts[40]. Subsequent studies have shown that the initial mutations in the mtDNA induced by UVR render the mitochondria in the fibroblasts more susceptible to ROS damage due to an increase in common deletions and a persistence of the mutations event after the termination of UVR exposure[41].

Langerhans cells are a subset of dendritic cells and are found in the epidermis of the skin. Langerhans cells play a role in the initiation and regulation of immune response by acquiring antigens in the skin and migrating to lymph nodes where immune responses are initiated. When human skin is exposed to UVR it results in a depletion of Langerhans cells and reduces the expression of immunophenotypic markers associated with antigen presentation[42]-[44]. The lack of functional Langerhans cells ultimately results in an immunosuppressive state in the skin[45] which also involves regulatory $\mathrm{T}$ cells and cytokine production[46].

\subsection{Mast cells}

It has been recognized that photoaging and normal chronological aging differ in several morphological and biological aspects, one of which is distinct alterations in elastic fibers and microvasculature. Previous studies have shown that one of the main contributors to this phenomenon is activation of immune cells in the epidermis, one of which is the mast cell. Studies comparing photo-exposed and sun-protected skin from the same individual shows that sun-exposed skin is characterized by the presence of higher numbers of infiltrating leucocytes and mast cells than is found in sun-protected skin[47]. Mast cells are cutaneous cells that contain pro-fibrotic and pro-inflammatory mediators and can contribute to skin inflammation and disease. Recent evidence has suggested that mast cells maintain tissue homeostasis and that many of the responses observed in photoaging are an attempt of the mast cell to repair damaged tissue systems. Mast cell activation can initiate or exacerbate many of the alterations associated with chronically sun-exposed skin such as massive accumulation of abnormal elastic fibers, loss of collagen, increase in glycosaminoglycans and telangiectatic vessels[47]. For example, mast cells produce fibroblast growth factors that can activate fibroblasts to produce abnormal matrix molecules. Mast cells also produce matrix-degrading enzymes and can activate the production of matrix proteinases by other skin cells such as keratinocytes. Mast cell products can recruit and activate infiltrating inflammatory cells such as neutrophils, macrophages and lymphocytes that can further damage the local tissue (see Figure 4).

Although an increased number of mast cells have been associated with photoaging, very little is known about their role in this process. The interaction between mast cells and other skin cells such as keratinocytes in photoaging is poorly understood. In fact, the precise mechanisms of mast cell activation in photodamaged skin is not known although it does not appear to be through the more common pathways such as the high affinity IgE receptor (FceRI) or pathogen-associated pattern recognition receptors. In photoaging, mast cell acti- 


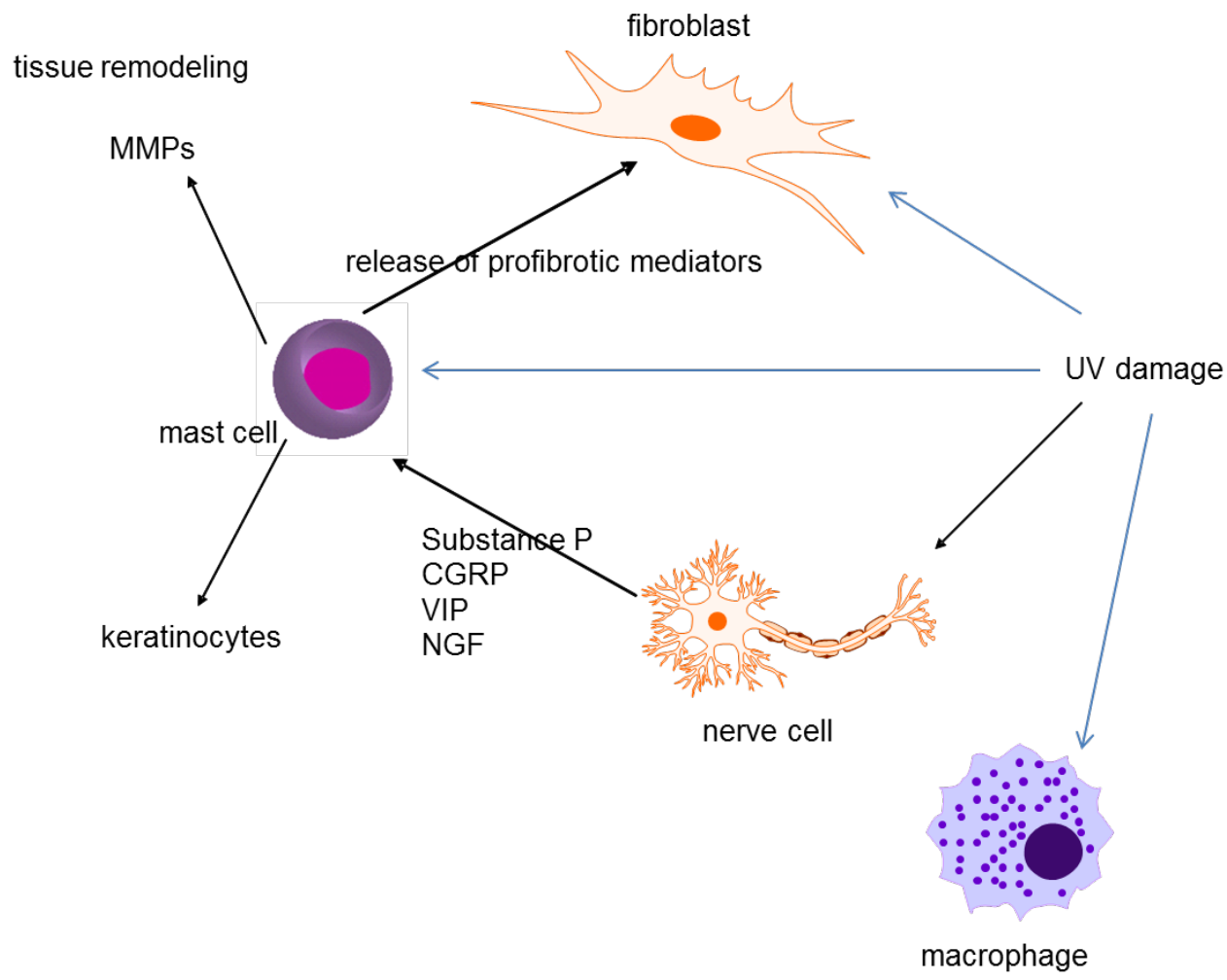

Figure 4. Role of skin cells in photoaging.

vation appears to be unique but our laboratory has shown that $G$ protein coupled receptors (GPCR) may be responsible. Chronic exposure to UV causes sensory nerve fibers to release a series of neuropeptides including substance $\mathrm{P}(\mathrm{SP})$ and calcitonin gene-related peptide (CGRP) [48], both of which can activate mast cell degranulation and pro-inflammatory mediator release[49]. Furthermore, opiates that modify nerve function can also activate skin mast cells[50]. Interestingly, SP causes mast cells to downregulate their expression of allergen-activated receptors (FceRI)[51] and primes them to respond to pathogen-activated (TLR) stimuli[52]. In skin that is chronically exposed to UV, mast cells may modulate a chronic inflammatory state, thus causing further damage to skin's structural integrity. Furthermore, mast cells appear to be important in the immunosuppressive environment of photodamaged skin since UVB causes a mast cell-dependent suppression of contact hypersensitivity responses[53]. This finding supports our hypothesis that human mast cells exposed to UV may undergo a phenotypic change from an allergic inflammatory effector cell to a more pathogen/structural response cell capable orchestrating structural changes in the skin. However, it is also possible that mast cell activation is a protective phenomenon and that further 
potentiating mast cell activation in photodamaged skin may, in fact, inhibit or reverse some of the immunosuppression associated with photodamage.

\subsection{Inflammation during photodamage}

Traditionally, photodamage has been characterized by the mechanical damage caused to the skin's constituents - i.e. the degradation of the proteins and structures that hold the skin together and give its integrity. However, recent evidence has shown that at least some of the damage associated with UV exposure is due to the initiation of low but chronic inflammation that ultimately results in impaired cellular responses. This has some major implications because it suggests that the skin is not only losing its ability to form a physical barrier but is also losing some of its immune and regenerative abilities. Recent studies have shown that photodamage reduces immune responses by inhibiting the pathways that activate immune cells and causes changes in biochemical processes that regulate wound healing.

Using histological analysis, Bosset et al showed that sun-exposed skin had an increase in infiltrating mononuclear cells than sun-protected skin[54]-[56]. Immunohistochemical analysis also showed an increased number of cells that one would normally associate with a Th2 inflammatory response - namely, mast cells, macrophages and CD4+ CD45RO+ T cells -- in sun-exposed dermis as well as a higher number of CD1a+ dendritic cells in sun-exposed epidermis, compared with the sun-protected samples. Although Bosset et al. found that photoaging displayed histological features of chronic skin inflammation they found a decreased expression of interleukin-1beta mRNA in sun-exposed compared with sun-protected suggesting that other pro-inflammatory mediators were mediating these effects. This hypothesis seems likely since these patients' skin looked clinically normal and would suggest an inflammatory pathology that is very different from those normally associated with acute Th2 inflammatory reactions such as atopic dermatitis or urticaria.

Inflammation is a complex process that has many different points of initiation and can be perpetuated by a diverse array of mediators. However, a study by Yan et al.[57] suggests that leukotrienes, potent lipid inflammatory mediators, may be responsible for this low but chronic inflammatory process associated with UV exposure. In their study, these investigators evaluated the protective effects of a nonsedative histamine H1-receptor antagonist, mizolastine, on UVB-exposed skin dermal fibroblasts. They found that relatively low levels of mizolastine $(10 \mathrm{nM})$ inhibited UVB-induced $\mathrm{LTB}_{4}$ production by skin fibroblasts. This decrease in $\mathrm{LTB}_{4}$ production was associated with a down-regulation of 5-lipooxygenase ( 5LO) mRNA expression and inhibition of 5-LO translocation suggesting that UV-induced leukotriene synthesis was dependent upon 5-LO expression.

The idea that chronic inflammation due to photodamage is a Th2 mediated response is possible, but some evidence suggests that it may be, in fact, a Th1 mediated event. UVB exposure of the skin results in a profound upregulation of the anti-inflammatory cytokine IL-10 and suppression of contact hypersensitivity (CHS). Furthermore, using an IL-10 transgenic mouse model (IL-10tg), Ma et al.[58] have shown that unexposed IL-10tg animals showed a diminished CHS response compared to wild-type. Yet when IL-10tg animals were exposed to UVB, the CHS response was not further suppressed, but rather was restored to the level 
observed in unexposed wild-type animals suggesting a normalization of the hypersensitivity response. If one believes that Th1 and Th2 responses counter-balance one another, this data would suggest that chronic inflammation associated with photodamage is mediated by a Th1 controlled inflammatory response.

\section{Natural ingredients as treatments for photodamage and photodamage}

\subsection{Targeting the skin's immune response}

Natural products that are used to treat the symptoms associated with photoaging and photodamage are designed to either stimulate new skin cell formation or inhibit the biochemical processes that cause skin damage. Sometimes, compounds are promoted as having biological activity in both of these contexts. Natural health products (NHPs) used to treat photodamaged skin are often mixtures of many different molecules and the precise mechanism of their beneficial effects are not always easy to determine from the myriad of information (anecdotal and experimental) available. However, there are a few groups of compounds that are naturally-derived and that appear to have some efficacy in at least reducing some of the biochemical manifestations of photodamage.

Botanical extracts are the most popular sources of naturally-sourced bioactives in skin health clinics. Most often, these are mixed into topical creams and applied to the epidermis. These extracts can even provide some small measure of sun protective activity (SPF) as well as reducing the biochemical and cellular consequences of photodamage described previously[59]. Topical application of green and white tea extracts, can reduce some of the detrimental immunomodulatory effects of photodamage[60]. Topical administration of Vitamin A and its analogues inhibit the expression of MMP and stimulate collagen synthesis in both photodamaged and photoprotected aged skin[61]. The list of these compounds is extensive and there are many comprehensive reviews on these compounds and their effects on human skin. However, these compounds all share the ability to modify the cellular responses in the dermis, whether through the induction of regenerative processes associated with keratinocyte renewal or inhibition of enzymes that break down the structural integrity of the skin. These compounds are all tested for their direct effect on these systems in bioassays designed to tease out the mechanisms on a single or small group of skin cells.

Some of these extracts, however, have efficacy when taken orally so their mechanisms of much more difficult to determine. For example, taking a specific Polypodium leucotomos extract (Fernblock, Cantabria Farmaceutica) $7.5 \mathrm{mg} / \mathrm{kg}$ daily for two doses before UVA treatment can modestly reduce erythema, edema, and signs of epidermal damage and may decrease subsequent hyperpigmentation in some patients[62]. In fact, it has been suggested that a diet rich in green tea polyphenols, grape seed proanthocyanidins and silymarin may act as chemopreventative agents to protect skin from photocarcinogenesis[63]. Taking what we know about the skin immune system and the possible molecular targets of these compounds we can hypothesize that this process may involve the induction of interleukin-12 (IL-12), DNA repair mechanisms, protection of Langerhans cell depletion and stimulation of cytotoxic $\mathrm{T}$ 
cells. However, many questions remain. How much of these chemoprotective agents must we eat and how often? Are some people (i.e. different skin tones) better protected by these dietary compounds than others? What are the best sources of these compounds in our diet? These are all relevant questions that are difficult to answer experimentally.

In addition to prophylactic treatments to alleviate the symptoms of photoaging, there is a strong market for compounds that can either stop or delay the sigs of photoaging. Anti-aging products are used to reduce the various cellular processes associated with senescence both intrinsic (chronoaging) and extrinsic (photoaging) ageing. Younger individuals seek to prevent or reduce the manifestations of the extrinsic aging process which is responsible for approximately 80 percent of facial aging. Older individuals are often concerned with preventing or reversing the signs of both intrinsic and extrinsic aging processes. As such, treatments used to prevent photoaging target the underlying inflammatory and molecular responses associated with damage of skin integrity. Treatments used to reverse or stop the appearance of photoaging often target the biomechanical properties of skin.

There are numerous compounds currently on the market and in development and it is beyond the scope of this chapter to cover all of them. However, alpha hydroxy acids serve as excellent example to illustrate their effects on the skin microenvironment. AHA include glycolic, citric, lactic, malic, tartaric and lactic acids and are used extensively to promote skin rejuvenation and renewal. They are sometimes marketed as "fruit acids" because citric acid is found in citric fruits, malic acid is present in apples and tartaric acid is sometimes isolated from grapes. However, other foods also contain these acids (sugarcane contains glycolic acid and sour milk contains lactic acid). AHA work mainly by exfoliating the stratum corneum thus improving the smoothness and texture of skin. Although the exact mechanism of this effect is unknown, it is believed that AHA inhibit the enzymes that form ionic sulfate and phosphate bonds in the stratum corneum[64]. In the dermis, AHA induce desquamation and promoting cell renewal as well as stimulate collagen synthesis and induce dermal thickness[65].

AHA peels have recently been recognized as important adjunctive therapy in a variety of conditions including photodamage, actinic damage, melasma, hyperpigmentation disorders, acne, and rosacea. Since AHA are considered to have a reasonable level of safety and efficacy in a variety of skin types, they have been used extensively in the cosmetic industry as well[66]. Although their exact mechanism of action is unknown, it has been suggested that AHA improve skin pathological disorders by thinning the stratum corneum, promoting epidermolysis, dispersing basal layer melanin, and increasing collagen synthesis within the dermis[67]. Specifically in patients with photodamaged skin, AHA peels and topical products are often combined with retinoids and other antioxidants to reduce the thickness of the stratum corneum and smooth out the skin's surface. It has been suggested that AHA may help increase the skin's barrier function, making it more resilient to chemical damage[68].

A new class of hydroxy acids called polyhydroxy acids (PHAs), have similar effects as AHA but irritate the skin the same way as classical AHAs. PHAs cam be used on clinically sensitive skin, including rosacea and atopic dermatitis, and are compatible with cosmetic procedures[69]. Compared to AHA, PHA formulations are more moisturizing and can enhance 
stratum corneum barrier function. PHAs such as gluconolactone or lactobionic acid may be used in combination with other products, ingredients, or procedures such as laser and microdermabrasion to provide additional benefits to therapy or to enhance the therapeutic effect. PHAs used in combination with retinyl acetate (pro-vitamin A) in a cream base can have some anti-aging skin benefits such as skin smoothing and plumping although it is unclear if these also involve biochemical or immunomodulatory effects. In some formulations, PHA and hydroquinone together have demonstrated some superficial improvement in morphological changes associated with photoaging and have resulted in a decrease in skin pigmentation.

\section{Conclusions}

The skin is a complex and dynamic organ that is constantly bombarded with environmental trauma including UV radiation. UV light can cause major and often irreversible changes in the skin including damage to the three layers of skin. In the context of this constantly changing and sometimes harsh environment, the skin must maintain homeostasis and protect a delicate network of biochemical and physiological systems. There are a number of processes that are designed to protect the skin from photodamage, including the stratum corneum, melanin and underlying immunological processes to control inflammation. However, it is becoming increasingly clear that, over time, the skin's protective mechanisms can become overwhelmed by the damage caused by UV radiation, resulting in irreversible structural damage, chronic inflammation and, in some cases, photocarcinogenesis. Understanding the role that the microenvironment plays in this complex process is important to developing innovative new strategies for its treatment and possibly reversal.

\section{Author details}

Marianna Kulka*

National Research Council, Canada

\section{References}

[1] Wakamatsu K, Nakanishi Y, Miyazaki N, Kolbe L, Ito S. UVA-induced oxidative degradation of melanins: fission of indole moiety in eumelanin and conversion to benzothiazole moiety in pheomelanin. Pigment cell \& melanoma research 2012; 25(4): 434-45. 
[2] Curto EV, Kwong C, Hermersdorfer H, et al. Inhibitors of mammalian melanocyte tyrosinase: in vitro comparisons of alkyl esters of gentisic acid with other putative inhibitors. Biochemical pharmacology 1999; 57(6): 663-72.

[3] Wrzesniok D, Beberok A, Otreba M, Buszman E. Modulation of melanogenesis and antioxidant defense system in melanocytes by amikacin. Toxicology in vitro : an international journal published in association with BIBRA 2013; 27(3): 1102-8.

[4] Tadokoro T, Yamaguchi Y, Batzer J, et al. Mechanisms of skin tanning in different racial/ethnic groups in response to ultraviolet radiation. The Journal of investigative dermatology 2005; 124(6): 1326-32.

[5] Aalborg J, Morelli JG, Mokrohisky ST, et al. Tanning and increased nevus development in very-light-skinned children without red hair. Archives of dermatology 2009; 145(9): 989-96.

[6] Langholz B, Richardson J, Rappaport E, Waisman J, Cockburn M, Mack T. Skin characteristics and risk of superficial spreading and nodular melanoma (United States). Cancer causes \& control : CCC 2000; 11(8): 741-50.

[7] Mitra D, Luo X, Morgan A, et al. An ultraviolet-radiation-independent pathway to melanoma carcinogenesis in the red hair/fair skin background. Nature 2012; 491(7424): 449-53.

[8] Gibney GT, Smalley KS. An unholy alliance: cooperation between BRAF and NF1 in melanoma development and BRAF inhibitor resistance. Cancer discovery 2013; 3(3): 260-3.

[9] Nagore E, Hacker E, Martorell-Calatayud A, et al. Prevalence of BRAF and NRAS mutations in fast-growing melanomas. Pigment cell \& melanoma research 2013; 26(3): 429-31.

[10] Kuntsche J, Bunjes H, Fahr A, et al. Interaction of lipid nanoparticles with human epidermis and an organotypic cell culture model. International journal of pharmaceutics 2008; 354(1-2): 180-95.

[11] Kiss B, Biro T, Czifra G, et al. Investigation of micronized titanium dioxide penetration in human skin xenografts and its effect on cellular functions of human skin-derived cells. Experimental dermatology 2008; 17(8): 659-67.

[12] Larese FF, D'Agostin F, Crosera M, et al. Human skin penetration of silver nanoparticles through intact and damaged skin. Toxicology 2009; 255(1-2): 33-7.

[13] Lee WR, Shen SC, Al-Suwayeh SA, Yang HH, Li YC, Fang JY. Skin permeation of small-molecule drugs, macromolecules, and nanoparticles mediated by a fractional carbon dioxide laser: the role of hair follicles. Pharmaceutical research 2013; 30(3): 792-802. 
[14] Abdel-Mottaleb MM, Moulari B, Beduneau A, Pellequer Y, Lamprecht A. Surfacecharge-dependent nanoparticles accumulation in inflamed skin. Journal of pharmaceutical sciences 2012; 101(11): 4231-9.

[15] Abdel-Mottaleb MM, Moulari B, Beduneau A, Pellequer Y, Lamprecht A. Nanoparticles enhance therapeutic outcome in inflamed skin therapy. European journal of pharmaceutics and biopharmaceutics : official journal of Arbeitsgemeinschaft fur Pharmazeutische Verfahrenstechnik eV 2012; 82(1): 151-7.

[16] Chourasia R, Jain SK. Drug targeting through pilosebaceous route. Current drug targets 2009; 10(10): 950-67.

[17] Seiji M, Fitzpatrick TB. The reciprocal relationship between melanization and tyrosinase activity in melanosomes (melanin granules). Journal of biochemistry 1961; 49: $700-6$.

[18] Kinnear PE, Jay B, Witkop CJ, Jr. Albinism. Survey of ophthalmology 1985; 30(2): 75-101.

[19] Hutton SM, Spritz RA. A comprehensive genetic study of autosomal recessive ocular albinism in Caucasian patients. Investigative ophthalmology \& visual science 2008; 49(3): 868-72.

[20] Koludrovic D, Davidson I. MITF, the Janus transcription factor of melanoma. Future oncology 2013; 9(2): 235-44.

[21] Wu XS, Masedunskas A, Weigert R, Copeland NG, Jenkins NA, Hammer JA. Melanoregulin regulates a shedding mechanism that drives melanosome transfer from melanocytes to keratinocytes. Proceedings of the National Academy of Sciences of the United States of America 2012; 109(31): E2101-9.

[22] Park SH, Kim DS, Kim WG, et al. Terrein: a new melanogenesis inhibitor and its mechanism. Cellular and molecular life sciences : CMLS 2004; 61(22): 2878-85.

[23] Kim HE, Ishihara A, Lee SG. The effects of Caffeoylserotonin on inhibition of melanogenesis through the downregulation of MITF via the reduction of intracellular cAMP and acceleration of ERK activation in B16 murine melanoma cells. BMB reports 2012; 45(12): 724-9.

[24] Park SH, Kim DS, Lee HK, et al. Long-term suppression of tyrosinase by terrein via tyrosinase degradation and its decreased expression. Experimental dermatology 2009; 18(6): 562-6.

[25] Jones K, Hughes J, Hong M, Jia Q, Orndorff S. Modulation of melanogenesis by aloesin: a competitive inhibitor of tyrosinase. Pigment cell research / sponsored by the European Society for Pigment Cell Research and the International Pigment Cell Society 2002; 15(5): 335-40. 
[26] Gillbro JM, Olsson MJ. The melanogenesis and mechanisms of skin-lightening agents--existing and new approaches. International journal of cosmetic science 2011; 33(3): 210-21.

[27] Solano F, Briganti S, Picardo M, Ghanem G. Hypopigmenting agents: an updated review on biological, chemical and clinical aspects. Pigment cell research / sponsored by the European Society for Pigment Cell Research and the International Pigment Cell Society 2006; 19(6): 550-71.

[28] Kim SY, Park KC, Kwon SB, Kim DS. Hypopigmentary effects of 4-n-butylresorcinol and resveratrol in combination. Die Pharmazie 2012; 67(6): 542-6.

[29] Fu B, Li H, Wang X, Lee FS, Cui S. Isolation and identification of flavonoids in licorice and a study of their inhibitory effects on tyrosinase. Journal of agricultural and food chemistry 2005; 53(19): 7408-14.

[30] Makino ET, Mehta RC, Banga A, Jain P, Sigler ML, Sonti S. Evaluation of a hydroquinone-free skin brightening product using in vitro inhibition of melanogenesis and clinical reduction of ultraviolet-induced hyperpigmentation. Journal of drugs in dermatology : JDD 2013; 12(3): s16.

[31] Chou TH, Ding HY, Hung WJ, Liang CH. Antioxidative characteristics and inhibition of alpha-melanocyte-stimulating hormone-stimulated melanogenesis of vanillin and vanillic acid from Origanum vulgare. Experimental dermatology 2010; 19(8): 742-50.

[32] Chung SY, Seo YK, Park JM, et al. Fermented rice bran downregulates MITF expression and leads to inhibition of alpha-MSH-induced melanogenesis in B16F1 melanoma. Bioscience, biotechnology, and biochemistry 2009; 73(8): 1704-10.

[33] Abdel-Malek ZA, Knittel J, Kadekaro AL, Swope VB, Starner R. The melanocortin 1 receptor and the UV response of human melanocytes--a shift in paradigm. Photochemistry and photobiology 2008; 84(2): 501-8.

[34] Hakozaki T, Minwalla L, Zhuang J, et al. The effect of niacinamide on reducing cutaneous pigmentation and suppression of melanosome transfer. The British journal of dermatology 2002; 147(1): 20-31.

[35] Leveque JL, Poelman MC, Legall F, de Rigal J. New experimental approach to measure the skin-reflected light. Application to cutaneous erythema and blanching. Dermatologica 1985; 170(1): 12-6.

[36] Berardesca E, Farage M, Maibach H. Sensitive skin: an overview. International journal of cosmetic science 2013; 35(1): 2-8.

[37] Fisher GJ, Datta SC, Talwar HS, et al. Molecular basis of sun-induced premature skin ageing and retinoid antagonism. Nature 1996; 379(6563): 335-9. 
[38] Szendroi M, Meimon G, Bakala H, et al. On the presence of a metalloprotease in human skin fibroblasts that degrades the human skin elastic fiber system. The Journal of investigative dermatology 1984; 83(3): 224-9.

[39] Sorrell JM, Caplan AI. Fibroblast heterogeneity: more than skin deep. Journal of cell science 2004; 117(Pt 5): 667-75.

[40] Berneburg M, Grether-Beck S, Kurten V, et al. Singlet oxygen mediates the UVA-induced generation of the photoaging-associated mitochondrial common deletion. The Journal of biological chemistry 1999; 274(22): 15345-9.

[41] Gremmel T, Wild S, Schuller W, et al. Six genes associated with the clinical phenotypes of individuals with deficient and proficient DNA repair. Translational oncogenomics 2008; 3: 1-13.

[42] Aberer W, Schuler G, Stingl G, Honigsmann H, Wolff K. Ultraviolet light depletes surface markers of Langerhans cells. The Journal of investigative dermatology 1981; 76(3): 202-10.

[43] Stingl G, Gazze-Stingl LA, Aberer W, Wolff K. Antigen presentation by murine epidermal langerhans cells and its alteration by ultraviolet B light. Journal of immunology 1981; 127(4): 1707-13.

[44] Aberer W, Leibl H. Effect of UVB radiation on the biosynthesis of HLA-DR antigens. Archives of dermatological research 1987; 279(5): 321-6.

[45] Schwarz A, Noordegraaf M, Maeda A, Torii K, Clausen BE, Schwarz T. Langerhans cells are required for UVR-induced immunosuppression. The Journal of investigative dermatology 2010; 130(5): 1419-27.

[46] Schwarz T, Schwarz A. Molecular mechanisms of ultraviolet radiation-induced immunosuppression. European journal of cell biology 2011; 90(6-7): 560-4.

[47] Lavker RM. Structural alterations in exposed and unexposed aged skin. The Journal of investigative dermatology 1979; 73(1): 59-66.

[48] Eschenfelder CC, Benrath J, Zimmermann M, Gillardon F. Involvement of substance $\mathrm{P}$ in ultraviolet irradiation-induced inflammation in rat skin. The European journal of neuroscience 1995; 7(7): 1520-6.

[49] Kulka M, Sheen CH, Tancowny BP, Grammer LC, Schleimer RP. Neuropeptides activate human mast cell degranulation and chemokine production. Immunology 2008; 123(3): 398-410.

[50] Sheen CH, Schleimer RP, Kulka M. Codeine induces human mast cell chemokine and cytokine production: involvement of G-protein activation. Allergy 2007; 62(5): 532-8.

[51] McCary C, Tancowny BP, Catalli A, et al. Substance P downregulates expression of the high affinity IgE receptor (FcepsilonRI) by human mast cells. Journal of neuroimmunology 2010; 220(1-2): 17-24. 
[52] Tancowny BP, Karpov V, Schleimer RP, Kulka M. Substance P primes lipoteichoic acid- and Pam3CysSerLys4-mediated activation of human mast cells by up-regulating Toll-like receptor 2. Immunology 2010; 131(2): 220-30.

[53] Hart PH, Grimbaldeston MA, Swift GJ, Jaksic A, Noonan FP, Finlay-Jones JJ. Dermal mast cells determine susceptibility to ultraviolet B-induced systemic suppression of contact hypersensitivity responses in mice. The Journal of experimental medicine 1998; 187(12): 2045-53.

[54] Bosset S, Barre P, Chalon A, et al. Skin ageing: clinical and histopathologic study of permanent and reducible wrinkles. European journal of dermatology : EJD 2002; 12(3): 247-52.

[55] Bosset S, Bonnet-Duquennoy M, Barre P, et al. Photoageing shows histological features of chronic skin inflammation without clinical and molecular abnormalities. The British journal of dermatology 2003; 149(4): 826-35.

[56] Bosset S, Bonnet-Duquennoy M, Barre P, et al. Decreased expression of keratinocyte beta1 integrins in chronically sun-exposed skin in vivo. The British journal of dermatology 2003; 148(4): 770-8.

[57] Yan Y, Wang B, Zuo YG, Qu T. Inhibitory effects of mizolastine on ultraviolet B-induced leukotriene B4 production and 5-lipoxygenase expression in normal human dermal fibroblasts in vitro. Photochemistry and photobiology 2006; 82(3): 665-9.

[58] Ma LJ, Guzman EA, DeGuzman A, et al. Unexpected effects of UVB in IL-10 transgenic mice: normalization of contact hypersensitivity response. Archives of dermatological research 2006; 297(9): 417-20.

[59] Matsui MS, Hsia A, Miller JD, et al. Non-sunscreen photoprotection: antioxidants add value to a sunscreen. The journal of investigative dermatology Symposium proceedings / the Society for Investigative Dermatology, Inc [and] European Society for Dermatological Research 2009; 14(1): 56-9.

[60] Camouse MM, Domingo DS, Swain FR, et al. Topical application of green and white tea extracts provides protection from solar-simulated ultraviolet light in human skin. Experimental dermatology 2009; 18(6): 522-6.

[61] Offord EA, Gautier JC, Avanti O, et al. Photoprotective potential of lycopene, betacarotene, vitamin E, vitamin $\mathrm{C}$ and carnosic acid in UVA-irradiated human skin fibroblasts. Free radical biology \& medicine 2002; 32(12): 1293-303.

[62] Middelkamp-Hup MA, Pathak MA, Parrado C, et al. Oral Polypodium leucotomos extract decreases ultraviolet-induced damage of human skin. Journal of the American Academy of Dermatology 2004; 51(6): 910-8.

[63] Katiyar SK. UV-induced immune suppression and photocarcinogenesis: chemoprevention by dietary botanical agents. Cancer letters 2007; 255(1): 1-11. 
[64] Berardesca E, Distante F, Vignoli GP, Oresajo C, Green B. Alpha hydroxyacids modulate stratum corneum barrier function. The British journal of dermatology 1997; 137(6): 934-8.

[65] Inan S, Oztukcan S, Vatansever S, et al. Histopathological and ultrastructural effects of glycolic acid on rat skin. Acta histochemica 2006; 108(1): 37-47.

[66] Van Scott EJ, Yu RJ. Alpha hydroxy acids: procedures for use in clinical practice. Cutis; cutaneous medicine for the practitioner 1989; 43(3): 222-8.

[67] Fartasch M, Teal J, Menon GK. Mode of action of glycolic acid on human stratum corneum: ultrastructural and functional evaluation of the epidermal barrier. Archives of dermatological research 1997; 289(7): 404-9.

[68] Rawlings AV, Davies A, Carlomusto M, et al. Effect of lactic acid isomers on keratinocyte ceramide synthesis, stratum corneum lipid levels and stratum corneum barrier function. Archives of dermatological research 1996; 288(7): 383-90.

[69] Grimes PE, Green BA, Wildnauer RH, Edison BL. The use of polyhydroxy acids (PHAs) in photoaged skin. Cutis; cutaneous medicine for the practitioner 2004; 73(2 Suppl): 3-13. 